Original Article

\title{
Review of the inquilinous fauna associated with insect galls in Brazilian restingas
}

\author{
Revisão da fauna inquilina associada a galhas de insetos em restingas brasileiras \\ V. C. Maia ${ }^{a *}$ (D) \\ ${ }^{a}$ Universidade Federal do Rio de Janeiro - UFRJ, Museu Nacional, Departamento de Entomologia, Rio de Janeiro, RJ, Brasil
}

\begin{abstract}
Several records of associated fauna, including parasitoids, inquilines, predators, and successors, have been reported by insect gall inventories in Brazilian restingas. Although most guilds are well established, inquilines have frequently been misinterpreted. In this paper, the inquilinous fauna of insect galls is revised based on five criteria: food habit; coexistence with the inducer; modification of gall tissues or production of new tissues; phylogenetic relationship with the inducer; and mobility. Gall inventories dated from 1988 to 2019 were examined, totaling 16 publications, eight of them with inquiline records. This guild was reported in 53 gall morphotypes in 44 plant species and four morphospecies distributed among 36 genera of 24 host families for a total of 65 records. Most inquilines were repositioned into the cecidophage guild and others into the kleptoparasite guild, resulting in a large reduction in the frequency of inquilines (from 65 to five records), and in first reports of cecidophages and kleptoparasites, with 46 and 13 records, respectively. Cecidophage was the most diverse guild with insects of five orders (Diptera, Coleoptera, Lepidoptera, Hemiptera, and Thysanoptera) while kleptoparasites were represented only by two orders (Diptera and Hymenoptera) and inquiline solely by Hymenoptera. Other results indicate that Leptothorax sp. (Formicidae) could be a successor and not an inquiline.
\end{abstract}

Keywords: Cecidophages, kleptoparasites, gall-inducing insects, Atlantic Forest.

\section{Resumo}

Vários registros de fauna associada, incluindo parasitoides, inquilinos, predadores e sucessores são encontrados em inventários de galhas de insetos em restingas brasileiras. Embora a maioria das guildas esteja bem estabelecida, os inquilinos são frequentemente interpretados de forma equivocada. Nesse trabalho, a fauna inquilina de galhas de insetos é revisada com base em cinco critérios: hábito alimentar, coexistência com o indutor, modificação dos tecidos da galha ou produção de novos tecidos, relação filogenética com o indutor e mobilidade. Inventários de galhas publicados entre 1988 e 2019 foram examinados, totalizando 16 artigos, oito deles com registro de inquilinos. Essa guilda foi assinalada em 53 morfotipos de galhas em 44 espécies de plantas e quatro morfoespécies distribuídas em 36 gêneros de 24 famílias vegetais, totalizando 65 registros. A maioria dos inquilinos foi reposicionada na guilda dos cecidófagos e outros na guilda dos cleptoparasitas, resultando em uma grande redução da frequência dos inquilinos (de 65 para cinco registros), e na primeira ocorrência de cecidófagos e cleptoparasitas, com 46 e 13 registros, respectivamente. A guilda dos cecidófagos foi a mais diversa, com insetos de cinco ordens (Diptera, Coleoptera, Lepidoptera, Hemiptera e Thysanoptera), enquanto que os cleptoparasitas foram representados por apenas duas ordens (Diptera e Hymenoptera) e os inquilinos somente por Hymenoptera. Outros resultados indicam que Leptothorax sp. (Formicidae) pode ser um sucessor e não um inquilino.

Palavras-chave: Cecidófagos, cleptoparasitas, insetos indutores de galhas, Mata Atlântica.

\section{Introduction}

Data on the arthropod fauna associated with insect galls have been reported by several inventories in five phytogeographic domains in Brazil: (1) Amazon Forest (Maia, 2011; Carvalho and Motta, 2018), (2) Atlantic Forest (Maia, 2001, 2013, 2014; Maia et al., 2008, 2014; Bregonci et al., 2010; Rodrigues et al., 2014; Maia and Souza, 2013; Carvalho-Fernandes et al., 2016; Maia and Carvalho-Fernandes, 2016; Maia and Mascarenhas, 2017;

Ansaloni et al., 2018; Flor et al., 2018; Maia and Siqueira, 2020), (3) Caatinga (Carvalho-Fernandes et al., 2012; Costa et al., 2014; Brito et al., 2018), (4) Cerrado (Fernandes et al., 1988; Urso-Guimarães et al., 2003; Maia and Fernandes, 2004; Araújo et al., 2007; Santos et al., 2010; Araújo et al., 2011; Saito and Urso-Guimarães, 2012; Santos et al., 2012, 2018; Bergamini et al., 2017; Lima and Calado, 2018; Silva et al., 2018 a, b; Vieira et al., 2018;

*e-mail: maiavcid@acd.ufrj.br

Received: March 21, 2020 - Accepted: October 8, 2020 
Ribeiro et al., 2019), and (5) Pantanal (Ascendino and Maia, 2018). Several gall inventories of Atlantic Forest areas were carried out in restingas, mainly in the state of Rio de Janeiro (Maia, 2001; Rodrigues et al., 2014; Carvalho-Fernandes et al., 2016), but also in Espírito Santo (Bregonci et al., 2010), and São Paulo (Maia et al., 2008). This fauna has been classified as parasitoids, inquilines, gall modifiers, successors, and predators. Nevertheless, other guilds are known, such as cecidophages, symbionts and kleptoparasites, but they were not been cited in these publications, probably due to terminological problems.

Mani (1964) defined most of these guilds. According to this author, successors are organisms that use the gall after the inducer leaves. They are mostly mites, spiders, thrips, beetles, ants and springtails, which apparently use gall tissues for food and shelter, but dead or decaying gall tissue is probably attacked by fungi that also serve as food. Inquilines and cecidophages use galls simultaneously with the galler. Both are phytophagous and consume gall tissues, but only the former promote the production of new tissues (they have been referred to as "gall modifiers" in some inventories). Kleptoparasites steal the resource (in this case the gall) from other organism, causing the galler death or its expulsion from the gall (Mound and Morris, 2000). Predators invade galls and feed on their inducers, killing them immediately, such as some species of Carabidae and Coccinellidae, for example (Ramamurthy, 2007). Parasitoids are organisms that live in close association with the host at the host's expense, which results in its death when the parasitoids complete their development (Godfray, 1994).

Guilds of predators, parasitoids, and successors are well delimited in the literature, while inquilines are a major problem as historically all organisms associated with galls that were not predators or parasitoids were placed in this guild (Redfern and Askew, 1992). Mani (1964), Godfray (1994), and Mound and Morris (2000) provided tools for distinguishing among these guilds, but none of them used multiple non-superposed parameters.

Luz and Mendonça-Júnior (2017) proposed five interaction parameters to conceptualize and differentiate inquilines, cecidophages and kleptoparasites from each other: food habit; coexistence with the inducer; modification of gall tissues or production of new tissues; phylogenetic relationship with the inducer; and mobility. According to these authors, inquilines are exclusively phytophagous, coexist with the gall-inducer, modify gall tissues or stimulate production of new tissues, have a close phylogenetic relationship with the inducer and are sedentary. Cecidophages are exclusively phytophagous, coexist with the gall-inducer, but do not modify gall tissues or stimulate production of new tissues, do not have a close phylogenetic relationship with the inducer and have high mobility, while kleptoparasites are omnivorous, do not coexist with the inducer, do not modify gall tissues or stimulate production of new tissues, can have a close phylogenetic relationship with the inducer and have low mobility. Based on these criteria, the inquilinous fauna associated with insect galls in Brazilian restingas is reviewed herein.
This paper also aims to answer the following questions: (1) Which are the most frequent and richest taxa of inquilines, cecidophages, and kleptoparasites? (2) Which gall-inducing taxa host the richest fauna of inquilines, cecidophages, and kleptoparasites? (3) In how many gall morphotypes and plant species, genera and families have these guilds been recorded? (4) Which plant families, genera and species shelter the richest guilds of inquilines, cecidophages, and kleptoparasites? (5) What are the most frequent features of host galls? (6) What is known about the taxonomy of the inquilines, cecidophages, and kleptoparasites of gall? and (7) What is necessary for the correct positioning of the associated fauna in the different guilds?

\section{Material and Methods}

A survey of scientific papers involving Brazilian restingas published from 1988 to 2019 was carried out by consulting the database "Web of Science" using "gall" or "galha" and "restinga" as keywords. Sixteen publications about galls in Brazilian restingas were found and examined. Eight of the publications provided data on inquilinous fauna, which were retrieved, compiled and analyzed, following the criteria established by Luz and Mendonça-Júnior (2017). The new positioning of associated fauna among guilds was based on literature data, as well as on unpublished biological information obtained from laboratory works, including gall arthropod rearing, gall dissection and observation of gall tissues and gall dweller behavior. These laboratory works were carried out during inventories of which I have participated, namely Maia, 2001; Maia et al., 2008; Bregonci et al., 2010; and Rodrigues et al. 2014. Finally, the positioning of the associated fauna into parasitoid and predator guilds was not revised, since the conceptions of these guilds are well understood.

\section{Results}

\subsection{Literature data}

Eight publications provided data on inquilinous fauna: Bregonci et al. (2010), Maia (1995, 2001, 2013), Maia and Azevedo (2009), Maia et al. (2002, 2008), and Rodrigues et al. (2014). However, this guild was misinterpreted, since it also comprised cecidophages and kleptoparasites, without discriminating them. Diptera (Cecidomyiidae, Chloropidae, Muscomorpha, Sciaridae, and Tephritidae), Coleoptera, Hemiptera (Aphidae, Coccidae, and Tingidae), Hymenoptera (Eulophidae, Formicidae and Tanaostigmatidae), Lepidoptera, and Thysanoptera were represented among the taxa indicated as inquilines by these authors (Table 1), with Diptera, Lepidoptera, and Coleoptera being the most frequent (Table 2 ). This secondary fauna was found in 53 gall morphotypes and totaled 65 records in 44 plant species and four morphospecies distributed among 36 genera of 24 host families. Myrtaceae was the family with the greatest number of records (Table 3 ). Most inquilines were positioned in other guilds, as shown below. 
Table 1. Inquilines found in insect gall inventories in Brazilian restingas published from 1988 to 2019.

\begin{tabular}{|c|c|c|c|c|}
\hline \multicolumn{2}{|c|}{ Host Plant } & \multirow{2}{*}{ Gall-inducer } & \multirow{2}{*}{ Inquiline } & \multirow{2}{*}{ Reference } \\
\hline Family & Species & & & \\
\hline Anacardiaceae & Atronium sp. & Hemiptera & $\begin{array}{l}\text { Clinodiplosis sp. } \\
\text { (Cecidomyiidae) }\end{array}$ & Maia (2013) \\
\hline \multirow[t]{8}{*}{ Asteraceae } & \multirow[t]{3}{*}{ Mikania cf. biformis DC. } & $\begin{array}{c}\text { Mikaniadiplosis } \\
\text { annulipes Gagné, } 2001 \\
\text { (Cecidomyiidae) }\end{array}$ & $\begin{array}{l}\text { Contarinia ubiquata Gagné, } \\
2001 \text { (Cecidomyiidae) }\end{array}$ & $\begin{array}{l}\text { Maia et al. } \\
\text { (2008) }\end{array}$ \\
\hline & & $\begin{array}{l}\text { Liodiplosis conica Gagné, } \\
2001 \text { (Cecidomyiidae) }\end{array}$ & $\begin{array}{c}\text { Trotteria sp. } \\
\text { (Cecidomyiidae) }\end{array}$ & $\begin{array}{l}\text { Maia et al. } \\
\text { (2008) }\end{array}$ \\
\hline & & $\begin{array}{l}\text { Liodiplosis conica Gagné, } \\
2001 \text { (Cecidomyiidae) }\end{array}$ & $\begin{array}{l}\text { Acanthocheilla sp. } \\
\text { (Tingidae) Aphididae and } \\
\text { Coccidae (Hemiptera), } \\
\text { Curculionidae (Coleoptera), }\end{array}$ & \\
\hline & $\begin{array}{l}\text { Baccharis singularis (Vell.) } \\
\text { G. M. Barroso }\end{array}$ & Neolasioptera sp. & Thysanoptera & $\begin{array}{l}\text { Maia et al. } \\
\text { (2008) }\end{array}$ \\
\hline & Baccharis speciosa DC. & Alycaulini (Cecidomyiidae) & Lepidoptera & $\begin{array}{l}\text { Maia et al. } \\
(2008)\end{array}$ \\
\hline & \multirow[t]{2}{*}{$\begin{array}{c}\text { Piptocarpha cf. cinerea } \\
\text { Baker }\end{array}$} & $\begin{array}{l}\text { Cecidomyiidi } \\
\text { (Cecidomyiidae) }\end{array}$ & Lepidoptera & $\begin{array}{l}\text { Maia et al. } \\
\text { (2008) }\end{array}$ \\
\hline & & Asphondylia sp. & $\begin{array}{c}\text { Lepidoptera and } \\
\text { Curculionidae (Coleoptera) }\end{array}$ & $\begin{array}{l}\text { Maia et al. } \\
\text { (2008) }\end{array}$ \\
\hline & $\begin{array}{l}\text { Porophyllum ruderale } \\
\text { (Jack.) Cass. }\end{array}$ & Asphondylia sp. & $\begin{array}{l}\text { Trypanea sp. (Diptera, } \\
\text { Tephritidae) }\end{array}$ & $\begin{array}{l}\text { Rodrigues et al. } \\
\qquad(2014)\end{array}$ \\
\hline Bignoniaceae & $\begin{array}{l}\text { Parabignonia unguiculata } \\
\text { (Vell.) A. H. Gentry }\end{array}$ & Cecidomyiidae & $\begin{array}{l}\text { Resseliella sp. } \\
\text { (Cecidomyiidae) }\end{array}$ & $\begin{array}{l}\text { Maia et al. } \\
\text { (2008) }\end{array}$ \\
\hline Boraginaceae & Varronia curassavica Jacq. & Lopesiini (Cecidomyiidae) & Curculionidae (Coleoptera) & $\begin{array}{l}\text { Maia et al. } \\
\text { (2008) }\end{array}$ \\
\hline Calophyllaceae & $\begin{array}{c}\text { Calophyllum brasiliense } \\
\text { Cambess. }\end{array}$ & $\begin{array}{c}\text { Lopesia elliptica Maia, } 2003 \\
\text { (Cecidomyiidae) }\end{array}$ & Coleoptera & $\begin{array}{l}\text { Maia et al. } \\
\text { (2008) }\end{array}$ \\
\hline Celastraceae & $\begin{array}{l}\text { Elachyptera micrantha } \\
\text { (Cambess.) A. C. Sm. }\end{array}$ & Cecidomyiidae & Lepidoptera & $\begin{array}{l}\text { Maia et al. } \\
\text { (2008) }\end{array}$ \\
\hline Erythroxylaceae & $\begin{array}{c}\text { Erythroxylum ovalifolium } \\
\text { Peyrs }\end{array}$ & $\begin{array}{c}\text { Lopesia erythroxyli } \\
\text { Rodrigues and Maia, } 2010\end{array}$ & Eulophidae (gall modifier) & Maia (2001 \\
\hline Euphorbiaceae & Croton compressus Lam. & Not determined & Curculionidae (Coleoptera) & $\begin{array}{l}\text { Rodrigues et al. } \\
\qquad(2014)\end{array}$ \\
\hline \multirow[t]{5}{*}{ Fabaceae } & \multirow[t]{2}{*}{ Andira fraxinifolia Benth. } & $\begin{array}{l}\text { Lopesia indaiensis } \\
\text { Maia \& Oliveira, } 2018\end{array}$ & $\begin{array}{l}\text { Curculionidae (Coleoptera), } \\
\text { Lepidoptera and } \\
\text { Cecidomyiidae }\end{array}$ & $\begin{array}{c}\text { Maia et al. } \\
\text { (2008), } \\
\text { Rodrigues et al. } \\
\text { (2014) }\end{array}$ \\
\hline & & Asphondyliina & Curculionidae (Coleoptera) & $\begin{array}{l}\text { Maia et al. } \\
\text { (2008) }\end{array}$ \\
\hline & $\begin{array}{l}\text { Andira nitida Mart. ex } \\
\text { Benth. }\end{array}$ & Cecidomyiidae & Coleoptera & $\begin{array}{l}\text { Bregonci et al. } \\
\text { (2010) }\end{array}$ \\
\hline & Inga laurina (Sw.) Willd. & $\begin{array}{c}\text { Meunieriella sp. } \\
\text { (Cecidomyiidae, Diptera) }\end{array}$ & $\begin{array}{l}\text { Tanaostigmatidae } \\
\text { (Hymenoptera) }\end{array}$ & $\begin{array}{l}\text { Maia et al. } \\
\text { (2002) }\end{array}$ \\
\hline & Inga sp. & $\begin{array}{l}\text { Neolasioptera sp. } \\
\text { (Cecidomyiidae) }\end{array}$ & Lepidoptera & $\begin{array}{l}\text { Rodrigues et al. } \\
\qquad(2014)\end{array}$ \\
\hline Lamiaceae & Hyptis fasciculata Benth. & Cecidomyiidae & Curculionidae (Coleoptera) & $\begin{array}{l}\text { Maia et al. } \\
\text { (2008) }\end{array}$ \\
\hline \multirow[t]{2}{*}{ Lauraceae } & $\begin{array}{l}\text { Ocotea lobbii (Meisn.) } \\
\text { Rohwer }\end{array}$ & Not determined & $\begin{array}{l}\text { Trotteria sp. } \\
\text { (Cecidomyiidae) }\end{array}$ & $\begin{array}{l}\text { Maia et al. } \\
\text { (2008) }\end{array}$ \\
\hline & $\begin{array}{c}\text { Ocotea notata } \\
\text { (Nees \& Mart.) Mez }\end{array}$ & Hemiptera & Cecidomyiidae & $\begin{array}{l}\text { Bregonci et al. } \\
\text { (2010) }\end{array}$ \\
\hline
\end{tabular}


Table 1. Continued..

\begin{tabular}{|c|c|c|c|c|}
\hline \multicolumn{2}{|c|}{ Host Plant } & \multirow{2}{*}{ Gall-inducer } & \multirow{2}{*}{ Inquiline } & \multirow{2}{*}{ Reference } \\
\hline Family & Species & & & \\
\hline & $\begin{array}{c}\text { Ocotea pulchella (Nees) } \\
\text { Mez }\end{array}$ & Cecidomyiidae & Hemiptera & $\begin{array}{l}\text { Maia et al. } \\
(2008)\end{array}$ \\
\hline & & Not determined & $\begin{array}{c}\text { Trotteria sp. } \\
\text { (Cecidomyiidae) }\end{array}$ & $\begin{array}{l}\text { Maia et al. } \\
\text { (2008) }\end{array}$ \\
\hline Loranthaceae & $\begin{array}{c}\text { Struthanthus concinnus } \\
\text { Mart. }\end{array}$ & $\begin{array}{l}\text { Asphondylia sp. } \\
\text { (Cecidomyiidae) }\end{array}$ & $\begin{array}{l}\text { Cecidomyiidae and } \\
\text { Thysanoptera }\end{array}$ & $\begin{array}{l}\text { Rodrigues et al. } \\
\qquad(2014)\end{array}$ \\
\hline Malvaceae & Luehea divaricata Mart & Cecidomyiidae & $\begin{array}{l}\text { Olcella sp. (Chloropidae, } \\
\text { Diptera) }\end{array}$ & $\begin{array}{l}\text { Rodrigues et al. } \\
\quad \text { (2014) }\end{array}$ \\
\hline \multirow[t]{2}{*}{ Melastomataceae } & $\begin{array}{l}\text { Miconia cinnamomifolia } \\
\text { (DC.) Naudin. }\end{array}$ & $\begin{array}{c}\text { Epihormomyia } \\
\text { miconiae Maia, 2001 } \\
\text { (Cecidomyiidae) }\end{array}$ & $\begin{array}{l}\text { Resseliella sp. } \\
\text { (Cecidomyiidae) }\end{array}$ & Maia (2001) \\
\hline & $\begin{array}{l}\text { Tibouchina trichopoda (DC.) } \\
\text { Baill. }\end{array}$ & Cecidomyiidae & Curculionidae (Coleoptera) & $\begin{array}{l}\text { Maia et al. } \\
(2008)\end{array}$ \\
\hline \multirow[t]{10}{*}{ Myrtaceae } & $\begin{array}{l}\text { Campomanesia guaviroba } \\
\text { (DC.) Kiaersk. }\end{array}$ & $\begin{array}{l}\text { Clinodiplosis sp. } \\
\text { (Cecidomyiidae) }\end{array}$ & Membracidae (Hemiptera) & $\begin{array}{l}\text { Maia et al. } \\
\text { (2008) }\end{array}$ \\
\hline & $\begin{array}{l}\text { Eugenia astringens } \\
\text { Cambess. }\end{array}$ & $\begin{array}{c}\text { Stephomyia rotundifoliorum } \\
\text { Maia, } 1994\end{array}$ & Eulophidae (gall modifier) & Maia (2001) \\
\hline & $\begin{array}{c}\text { Eugenia copacabanensis } \\
\text { Kiaersk. }\end{array}$ & $\begin{array}{c}\text { Stephomyia tetralobae } \\
\text { Maia, } 1994\end{array}$ & $\begin{array}{c}\text { Trotteria sp. } \\
\text { (Cecidomyiidae) }\end{array}$ & Maia (1995) \\
\hline & Eugenia hiemalis Cambess. & Stephomyia sp. & $\begin{array}{c}\text { Leptothorax sp. } \\
\text { (Formicidae, Hymenoptera) }\end{array}$ & Maia (2001) \\
\hline & $\begin{array}{c}\text { Eugenia punicifolia (Kunt) } \\
\text { DC. }\end{array}$ & Not determined & Curculionidae (Coleoptera) & $\begin{array}{l}\text { Rodrigues et al. } \\
\qquad(2014)\end{array}$ \\
\hline & Eugenia speciosa Cambess. & $\begin{array}{l}\text { Schizomyiina Clinodiplosis } \\
\text { sp. (Cecidomyiidae) }\end{array}$ & Sciaridae (Diptera) & $\begin{array}{l}\text { Maia et al. } \\
(2008)\end{array}$ \\
\hline & Myrcia ovata Cambess. & $\begin{array}{c}\text { Myrciamyia } \\
\text { maricaensis Maia, } 1995 \\
\text { (Cecidomyiidae) }\end{array}$ & $\begin{array}{c}\text { Eulophidae sp.1 (gall } \\
\text { modifier), Eulophidae sp. } 2 \\
\text { (endogaller) }\end{array}$ & $\begin{array}{l}\text { Ferraz and } \\
\text { Monteiro } \\
(2003)\end{array}$ \\
\hline & $\begin{array}{c}\text { Myrciaria floribunda } \\
\text { (H.West ex Willd.) O. Berg }\end{array}$ & Cecidomyiidae & Thysanoptera & $\begin{array}{l}\text { Bregonci et al. } \\
\text { (2010) }\end{array}$ \\
\hline & $\begin{array}{l}\text { Neomitranthes obscura } \\
\text { (DC.) N. J. E. Silveira }\end{array}$ & $\begin{array}{l}\text { Neomitranthella } \\
\text { robusta Maia, } 1996 \\
\text { (Cecidomyiidae) }\end{array}$ & $\begin{array}{l}\text { Stenoma annosa (Butler, } \\
\text { 1877) (Lepidoptera) }\end{array}$ & $\begin{array}{l}\text { Personnal } \\
\text { observation }\end{array}$ \\
\hline & & Cecidomyiidae & S.annosa & Maia (2001) \\
\hline \multirow[t]{4}{*}{ Nyctaginaceae } & $\begin{array}{c}\text { Guapira opposita (Vell.) } \\
\text { Reitz }\end{array}$ & $\begin{array}{l}\text { Bruggmannia elongata } \\
\text { Maia \& Couri, } 1993 \\
\text { (Cecidomyiidae) }\end{array}$ & Lepidoptera & $\begin{array}{l}\text { Maia et al. } \\
\text { (2008) }\end{array}$ \\
\hline & & $\begin{array}{l}\text { Bruggmannia sp. } \\
\text { (Cecidomyiidae) }\end{array}$ & Aphididae (Hemiptera) & $\begin{array}{l}\text { Rodrigues et al. } \\
\qquad(2014)\end{array}$ \\
\hline & & Pisphondylia sp. & Lepidoptera & $\begin{array}{l}\text { Rodrigues et al. } \\
\qquad(2014)\end{array}$ \\
\hline & $\begin{array}{l}\text { Guapira pernambucensis } \\
\text { (Casar.) Lundell }\end{array}$ & Cecidomyiidae & Lepidoptera & $\begin{array}{l}\text { Bregonci et al. } \\
\text { (2010) }\end{array}$ \\
\hline Ochnaceae & $\begin{array}{c}\text { Ouratea cuspidata (A.St.- } \\
\text { Hil.) Engl. }\end{array}$ & $\begin{array}{l}\text { Contarinia sp. } \\
\text { (Cecidomyiidae) }\end{array}$ & Coleoptera & $\begin{array}{l}\text { Bregonci et al. } \\
\text { (2010) }\end{array}$ \\
\hline Peraceae & $\begin{array}{c}\text { Chaetocarpus myrsinites } \\
\text { Baill. }\end{array}$ & Not determined & Lepidoptera & $\begin{array}{l}\text { Bregonci et al. } \\
\text { (2010) }\end{array}$ \\
\hline Polygalaceae & Securidaca sp. & Cecidomyiidae & Muscomorpha (Diptera) & $\begin{array}{l}\text { Rodrigues et al. } \\
\text { (2014) }\end{array}$ \\
\hline
\end{tabular}


Table 1. Continued..

\begin{tabular}{|c|c|c|c|c|}
\hline \multicolumn{2}{|c|}{ Host Plant } & \multirow{2}{*}{ Gall-inducer } & \multirow{2}{*}{ Inquiline } & \multirow{2}{*}{ Reference } \\
\hline Family & Species & & & \\
\hline Polygonaceae & Coccoloba alnifolia Casar & Lopesia sp. & Lepidoptera & $\begin{array}{l}\text { Rodrigues et al. } \\
\text { (2014) }\end{array}$ \\
\hline \multirow[t]{3}{*}{ Sapindaceae } & $\begin{array}{l}\text { Paullinia weinmanniifolia } \\
\text { Mart. }\end{array}$ & $\begin{array}{l}\text { Paulliniamyia ampla Maia, } \\
2001 \text { (Cecidomyiidae) }\end{array}$ & Eulophidae (endogaller) & Maia (2001) \\
\hline & Paullinia sp. & $\begin{array}{l}\text { Neolasioptera sp. } \\
\text { (Cecidomyiidae) }\end{array}$ & Lepidoptera & $\begin{array}{l}\text { Maia et al. } \\
(2008)\end{array}$ \\
\hline & $\begin{array}{l}\text { Serjania communis } \\
\text { Cambess. }\end{array}$ & $\begin{array}{l}\text { Clinodiplosis sp. } \\
\text { (Cecidomyiidae) }\end{array}$ & Sciaridae (Diptera) & $\begin{array}{l}\text { Maia et al. } \\
\text { (2008) }\end{array}$ \\
\hline \multirow[t]{5}{*}{ Sapotaceae } & $\begin{array}{c}\text { Pouteria venosa (Mart.) } \\
\text { Baehni }\end{array}$ & $\begin{array}{l}\text { Lopesia singularis Maia, } \\
2001 \text { (Cecidomyiidae) }\end{array}$ & Lepidoptera & $\begin{array}{l}\text { Maia et al. } \\
\text { (2008) }\end{array}$ \\
\hline & $\begin{array}{l}\text { Manilkara subsericea } \\
\text { (Mart.) Dubard }\end{array}$ & $\begin{array}{l}\text { Cecidomyiidi } \\
\text { (Cecidomyiidae) }\end{array}$ & $\begin{array}{l}\text { Contarinia sp. } \\
\text { (Cecidomyiidae) }\end{array}$ & Maia (2001) \\
\hline & & Cecidomyiidae & Coleoptera & $\begin{array}{l}\text { Bregonci et al. } \\
\text { (2010) }\end{array}$ \\
\hline & $\begin{array}{l}\text { Pouteria caimito } \\
\text { (Ruiz \& Pav.) Radlk. }\end{array}$ & $\begin{array}{c}\text { Youngomyia } \\
\text { pouteriae Maia, } 2001 \\
\text { (Cecidomyiidae) }\end{array}$ & $\begin{array}{c}\text { Trotteria quadridentata } \\
\text { Maia, } 2001 \\
\text { (Cecidomyiidae) }\end{array}$ & Maia (2013) \\
\hline & $\begin{array}{l}\text { Sideroxylon obtusifolium } \\
\text { (Roem. \& Schult.) T. D. } \\
\text { Penn. }\end{array}$ & $\begin{array}{l}\text { Bruggmannniella sideroxyli } \\
\text { Rodrigues \& Maia, } 2020\end{array}$ & Cecidomyiidae & $\begin{array}{l}\text { Rodrigues et al. } \\
\text { (2014) }\end{array}$ \\
\hline Solanaceae & $\begin{array}{c}\text { Aureliana fasciculata (Vell.) } \\
\text { Sendtn. }\end{array}$ & $\begin{array}{l}\text { Clinodiplosis sp. } \\
\text { (Cecidomyiidae) }\end{array}$ & Curculionidae (Coleoptera) & $\begin{array}{l}\text { Maia et al. } \\
\text { (2008) }\end{array}$ \\
\hline Verbenaceae & Stachytarpheta sp. & $\begin{array}{c}\text { Schizomyia stachytarphetae } \\
\text { Barnes, } 1932 \\
\text { (Cecidomyiidae) }\end{array}$ & $\begin{array}{l}\text { Haplothrips gowdeyi } \\
\text { (Franklin, 1908) } \\
\text { (Thysanoptera, } \\
\text { Phlaeothripidae) }\end{array}$ & $\begin{array}{l}\text { Rodrigues et al. } \\
\qquad(2014)\end{array}$ \\
\hline
\end{tabular}

\subsection{Recategorization (Table 4)}

\subsubsection{Cecidophage guild}

Although Coleoptera, Sciaridae, Tephritidae, Chloropidae, Muscomorpha, Clinodiplosis sp. (Cecidomyiidae), Hemiptera, Lepidoptera, and Thysanoptera were represented among the taxa considered inquilines, biological observations indicated these to be cecidophages since they feed on galls without modifying them or stimulating production of new tissues, coexisted with the inducer and had high mobility.

Other laboratory observations showed that immature stages of dipterans, coleopterans and lepidopterans occurred in galls, but not the adults, since they left galls immediately after their emergence. Different from these insects, adult hemipterans and thysanopterans were frequently observed in galls together with their eggs and nymphs. Pupal exuviae of coleopterans, lepidopterans, tephritids and chloropids were found in galls, while those of sciarids remained attached to gall openings, in the same way as cecidomyiid exuviae.

Field and laboratory observations showed that caterpillars, such as that of Stenoma annosa, for example, could be voracious, feeding and destroying several galls (Butler, 1877). Furthermore, their excrement is accumulated in the internal chamber(s).
These disturbances can lead to the death of the gallinducer, as previously reported (Maia, 2001).

Whenever larvae of coleopterans and lepidopterans were observed, the gall-inducer died, which did not happen when dipterans, hemipterans and thysanopterans were present.

The cecidophage guild was obtained from 39 gall morphotypes (75\%) on plants of 20 families (83\%), 28 genera (78\%) and 35 species (83\%) (Table 5 ). Asteraceae and Myrtaceae were the host families with the greatest number of records, with ten and six, respectively, which corresponded to about $21 \%$ and $13 \%$ of the total. However, in both families, the number of gall morphotypes (six) and plant species (five) that hosted cecidophages was nearly the same, corresponding to $15 \%$ and $14 \%$, respectively. Fabaceae totaled five records (11\%), three gall morphotypes (8\%), and three plant species (8\%), followed by Nyctaginaceae with four (9\%), four (10\%) and three (8\%), respectively.

Mikania Wild. (Asteraceae), Andira Juss. (Fabaceae), and Guapira Aubl. (Nyctaginaceae) were the plant genera with the highest number of records of cecidophages, with four each (9\%). Mikania cf. biformis DC., Andira fraxinifolia Benth. (Fabaceae), Guapira opposita (Vell.) Reitz (Nyctaginaceae), Piptocarpha cf. cinerea Baker, and Neomitranthes obscura (DC.) N. J. E. Silveira (Myrtaceae) were the plant species 
Table 2. Number of host species, gall morphotypes and records of inquilines by plant family in insect gall inventories in Brazilian restingas published from 1988 to 2019.

\begin{tabular}{|c|c|c|c|}
\hline \multicolumn{2}{|c|}{ Host Plant } & \multirow{2}{*}{ Number of gall morphotypes } & \multirow{2}{*}{ Number of records of inquilines } \\
\hline Family & Number of species & & \\
\hline Myrtaceae & 9 & 10 & 11 \\
\hline Asteraceae & 5 & 8 & 11 \\
\hline Fabaceae & 4 & 5 & 7 \\
\hline Sapotaceae & 4 & 5 & 5 \\
\hline Lauraceae & 3 & 4 & 4 \\
\hline Nyctaginaceae & 2 & 4 & 4 \\
\hline Sapindaceae & 3 & 3 & 3 \\
\hline Melastomataceae & 2 & 2 & 2 \\
\hline Anacardiaceae & 1 & 1 & 1 \\
\hline Bignoniaceae & 1 & 1 & 1 \\
\hline Boraginaceae & 1 & 1 & 1 \\
\hline Calophyllaceae & 1 & 1 & 1 \\
\hline Celastraceae & 1 & 1 & 1 \\
\hline Erythroxylaceae & 1 & 1 & 1 \\
\hline Euphorbiaceae & 1 & 1 & 1 \\
\hline Lamiaceae & 1 & 1 & 1 \\
\hline Loranthaceae & 1 & 1 & 2 \\
\hline Malvaceae & 1 & 1 & 1 \\
\hline Ochnaceae & 1 & 1 & 1 \\
\hline Peraceae & 1 & 1 & 1 \\
\hline Polygalaceae & 1 & 1 & 1 \\
\hline Polygonaceae & 1 & 1 & 1 \\
\hline Solanaceae & 1 & 1 & 1 \\
\hline Verbenaceae & 1 & 1 & 1 \\
\hline
\end{tabular}

Table 3. Frequency of inquilines in insect gall inventories in Brazilian restingas published from 1988 to 2019.

\begin{tabular}{ccc}
\hline Inquiline & Cecidomyiidae & $\begin{array}{c}\text { Number of } \\
\text { records }\end{array}$ \\
\hline Diptera & Sciaridae & 19 \\
& Chloropidae & 2 \\
& Muscomorpha & 1 \\
Lepidoptera & Tephritidae & 1 \\
Coleoptera & & 1 \\
Hymenoptera & & 15 \\
Thysanoptera & & 14 \\
Hemiptera & & 7 \\
\hline
\end{tabular}

with more than one cecidophage record. The first hosted aphidids, coccids, tingids (Hemiptera), and curculionids (Coleoptera) in only one gall morphotype; the second hosted curculionids and lepidopterans in one morphotype as well; the third hosted curculionids and lepidopterans in two morphotypes; and the last, hosted lepidopterans in two gall morphotypes.

Concerning plant organs, cecidophages were obtained from galls on leaves, stems, buds, tendrils, flowers, and fruits, with leaf galls being most frequent (61\%). They occurred in galls of several shapes, but mainly in globoid galls (31\%). Although they were obtained from green, brown, yellow, red, and purple galls, $67 \%$ occurred in green galls, and $95 \%$ occurred in glabrous galls.

Cecidophages represented five insect orders: Coleoptera, Diptera (Cecidomyiidae, Chloropidae, Muscomorpha, Sciaridae, Tephritidae), Hemiptera (Aphididae, Membracidae), Lepidoptera, and Thysanoptera. Among these, lepidopterans and coleopterans were the 
Table 4. Records of cecidophages, kleptoparasites, and inquilines in insect galls in Brazilian restingas.

\begin{tabular}{|c|c|c|}
\hline Host Plant & Gall-inducers & Associated fauna \\
\hline Atronium sp. & Hemiptera & Clinodiplosis sp. (cecidophage) \\
\hline \multirow[t]{2}{*}{ Mikania cf. biformis DC. } & Mikaniadiplosis annulipes Gagné, 2001 & $\begin{array}{c}\text { Contarinia ubiquita Gagné, } 2001 \\
\text { (kleptoparasite) }\end{array}$ \\
\hline & Liodiplosis conica Gagné. 2001 & Trotteria sp. (kleptoparasite) \\
\hline Baccharis singularis (Vell.) G. M. Barroso & Neolasioptera sp. & Thysanoptera (cecidophage) \\
\hline Baccharis speciosa DC. & Alycaulini & Lepidoptera (cecidophage) \\
\hline Piptocarpha cf. cinerea Baker & Cecidomyiidi & Lepidoptera (cecidophage) \\
\hline Porophyllum ruderale (Jack.) Cass. & Asphondylia sp. & Trypanea sp. (cecidophage) \\
\hline $\begin{array}{c}\text { Parabignonia unguiculata (Vell.) A. H. } \\
\text { Gentry }\end{array}$ & Cecidomyiidae & Resseliella sp. (kleptoparasite) \\
\hline Varronia curassavica Jacq. & Lopesiini & Curculionidae (cecidophage) \\
\hline Calophyllum brasiliense Cambess. & Lopesia elliptica Maia, 2003 & Coleoptera (cecidophage) \\
\hline $\begin{array}{l}\text { Elachyptera micrantha (Cambess.) A. C. } \\
\text { Sm. }\end{array}$ & Cecidomyiidae & Lepidoptera (cecidophage) \\
\hline Erythroxylum ovalifolium Peyrs. & $\begin{array}{l}\text { Lopesia erythroxyli Rodrigues and Maia, } \\
2010\end{array}$ & Eulophidae (inquiline) \\
\hline Croton compressus Lam. & Not determined & Curculionidae (cecidophage) \\
\hline \multirow[t]{4}{*}{ Andira fraxinifolia Benth. } & Cecidomyiidi & $\begin{array}{l}\text { Curculionidae and Lepidoptera } \\
\text { (cecidophages) }\end{array}$ \\
\hline & Asphondyliina & Curculionidae (cecidophage) \\
\hline & Lopesia indaiensis Maia \& Oliveira, 2018 & Cecidomyiidae (kleptoparasite) \\
\hline & Cecidomyiidae & Coleoptera (cecidophage) \\
\hline Inga laurina (Sw.) Willd. & Meunieriella sp. & Tanaostigmatidae (kleptoparasite) \\
\hline Inga sp. & Neolasioptera sp. & Lepidoptera (cecidophage) \\
\hline Hyptis fasciculata Benth. & Cecidomyiidae & Curculionidae (cecidophage) \\
\hline Ocotea lobbii (Meisn.) Rohwer & Not determined & Trotteria sp. (kleptoparasite) \\
\hline Ocotea notata (Nees \& Mart.) Mez & Hemiptera & Cecidomyiidae (cecidophage) \\
\hline \multirow[t]{2}{*}{ Ocotea pulchella (Nees) Mez } & Cecidomyiidae & Hemiptera (cecidophage) \\
\hline & Not determined & Trotteria sp. (kleptoparasite) \\
\hline Struthanthus concinnus Mart. & Asphondylia sp. & $\begin{array}{l}\text { Cecidomyiidae (kleptoparasite) and } \\
\text { Thysanoptera (cecidophage) }\end{array}$ \\
\hline Luehea divaricata Mart. & Cecidomyiidae & Olcella sp. (cecidophage) \\
\hline Miconia cinnamomifolia (DC.) Naudin. & Epihormomyia miconiae Maia, 2001 & Resseliella sp. (kleptoparasite) \\
\hline Tibouchina trichopoda (DC.) Baill. & Cecidomyiidae & Curculionidae (cecidophage) \\
\hline Campomanesia guaviroba (DC.) Kiaersk. & Clinodiplosis sp. & Membracidae (cecidophage) \\
\hline Eugenia astringens Cambess. & Stephomyia rotundifoliorum Maia, 1994 & Eulophidae (inquiline) \\
\hline Eugenia copacabanensis Kiaersk. & Stephomyia tetralobae Maia, 1994 & Trotteria sp. (kleptoparasite) \\
\hline Eugenia hiemalis Cambess. & Stephomyia sp. & Leptothorax sp. (successor) \\
\hline Eugenia punicifolia (Kunt) DC. & Not determined & Curculionidae (cecidophage) \\
\hline Eugenia speciosa Cambess. & Schizomyiina & Sciaridae (cecidophage) \\
\hline Myrcia ovata Cambess. & Myrciamyia maricaensis Maia, 1995 & $\begin{array}{l}\text { Eulophidae sp.1 and Eulophidae sp. } 2 \\
\text { (inquilines) }\end{array}$ \\
\hline $\begin{array}{c}\text { Myrciaria floribunda (H.West ex Willd.) } \\
\text { O. Berg }\end{array}$ & Cecidomyiidae & Thysanoptera (cecidophage) \\
\hline $\begin{array}{l}\text { Neomitranthes obscura (DC.) N. J. E. } \\
\text { Silveira }\end{array}$ & Neomitranthella robusta Maia, 2001 & $\begin{array}{c}\text { Stenoma annosa (Butler, 1877) } \\
\text { (cecidophage) }\end{array}$ \\
\hline
\end{tabular}


Table 4. Continued..

\begin{tabular}{|c|c|c|}
\hline Host Plant & Gall-inducers & Associated fauna \\
\hline & Clinodiplosini & Stenoma annosa (cecidophage) \\
\hline \multirow[t]{3}{*}{ Guapira opposita (Vell.) Reitz } & $\begin{array}{l}\text { Bruggmannia elongata Maia \& Couri, } \\
1993\end{array}$ & Lepidoptera (cecidophage) \\
\hline & Bruggmannia sp. & Aphididae (cecidophage) \\
\hline & Pisphondylia sp. & Lepidoptera (cecidophage) \\
\hline G. pernambucensis (Casar.) Lundell & Cecidomyiidae & Lepidoptera (cecidophage) \\
\hline Ouratea cuspidata (A.St.-Hil.) Engl. & Contarinia sp. & Coleoptera (cecidophage) \\
\hline Chaetocarpus myrsinites Baill. & Not determined & Lepidoptera (cecidophage) \\
\hline Securidaca sp. & Cecidomyiidae & Muscomorpha (cecidophage) \\
\hline Coccoloba alnifolia Casar & Lopesia sp. & Lepidoptera (cecidophage) \\
\hline Paullinia weinmanniifolia Mart. & Paulliniamyia ampla Maia, 2001 & Eulophidae (inquiline) \\
\hline Paullinia sp. & Neolasioptera sp. (Cecidomyiidae) & Lepidoptera (cecidophage) \\
\hline Serjania communis Cambess. & Clinodiplosis sp. & Sciaridae (cecidophage) \\
\hline Pouteria venosa (Mart.) Baehni & Lopesia singularis Maia, 2001 & Lepidoptera (cecidophage) \\
\hline Manilkara subsericea & Cecidomyiidi & Contarinia sp. (kleptoparasite) \\
\hline (Mart.) Dubard & Cecidomyiidae & Coleoptera (cecidophage) \\
\hline Pouteria caimito (Ruiz \& Pav.) Radlk. & Youngomyia pouteriae Maia, 2001 & $\begin{array}{l}\text { Trotteria quadridentata Maia, } 2001 \\
\text { (kleptoparasite) }\end{array}$ \\
\hline $\begin{array}{l}\text { Sideroxylon obtusifolium } \\
\text { (Roem. \& Schult.) T. D. }\end{array}$ & $\begin{array}{l}\text { Bruggmannniella sideroxyli } \\
\text { Rodrigues \& Maia, } 2020\end{array}$ & Cecidomyiidae (kleptoparasite) \\
\hline Aureliana fasciculata (Vell.) Sendtn. & Clinodiplosis sp. & Curculionidae (cecidophage) \\
\hline Stachytarpheta sp. & Schizomyia stachytarphetae Barnes, 1932 & $\begin{array}{l}\text { Haplothrips gowdeyi (Franklin, 1908) } \\
\text { (cecidophage) }\end{array}$ \\
\hline
\end{tabular}

Table 5. Cecidophages, kleptoparasites and inquilines found in gall inventories in Brazilian restingas and gall characterization.

\begin{tabular}{|c|c|c|c|c|c|}
\hline \multirow{2}{*}{$\begin{array}{c}\text { Guilds } \\
\text { Inquilines }\end{array}$} & \multirow{2}{*}{ Host plant } & \multicolumn{4}{|c|}{ Gall characterization } \\
\hline & & Host organ & Shape & Color & Trichomes \\
\hline Eulophidae & Erythroxylum ovalifolium Peyrs. & Bud & Conical & Green & Absent \\
\hline Eulophidae & Eugenia astringens Cambess. & Bud & Cylindrical & Brown & Absent \\
\hline Eulophidae sp.1 & Myrcia ovata Cambess. & Bud & Ovoid & Green & Absent \\
\hline Eulophidae sp. 2 & Myrcia ovata & Bud & Ovoid & Green & Absent \\
\hline Eulophidae & Paullinia weinmanniifolia Mart. & Leaf & Conical & Green & Absent \\
\hline \multicolumn{6}{|l|}{ Kleptoparasites } \\
\hline Cecidomyiidae & Andira fraxinifolia Benth. & Leaf & Vermiform & Green & Absent \\
\hline Cecidomyiidae & Struthanthus concinnus Mart. & Leaf and stem & Conical & Green & Absent \\
\hline Cecidomyiidae & $\begin{array}{l}\text { Sideroxylon obtusifolium } \\
\text { (Roem. \& Schult.) T. D. Penn. }\end{array}$ & Fruit & Globoid & Green & Absent \\
\hline $\begin{array}{l}\text { Contarinia } \\
\text { ubiquita }\end{array}$ & Mikania cf. biformis DC. & Leaf vein, petiole and stem & Fusiform & Green & Absent \\
\hline Contarinia sp. & $\begin{array}{c}\text { Manilkara subsericea (Mart.) } \\
\text { Dubard }\end{array}$ & Leaf & Lenticular & Green & Absent \\
\hline Resseliella sp. & $\begin{array}{c}\text { Parabignonia unguiculata (Vell.) } \\
\text { A. H. Gentry }\end{array}$ & Leaf & Lenticular & Green & Absent \\
\hline Resseliella sp. & $\begin{array}{l}\text { Miconia cinnamomifolia (DC.) } \\
\text { Naudin. }\end{array}$ & Bud & Ovoid & Green & Absent \\
\hline
\end{tabular}


Table 5. Continued..

\begin{tabular}{|c|c|c|c|c|c|}
\hline Guilds & \multirow{2}{*}{ Host plant } & \multicolumn{4}{|c|}{ Gall characterization } \\
\hline Inquilines & & Host organ & Shape & Color & Trichomes \\
\hline Tanaostigmatidae & Inga laurina (Sw.) Willd. & Leaf & Cylindrical & Yellow & Absent \\
\hline $\begin{array}{c}\text { Trotteria } \\
\text { quadridentata }\end{array}$ & $\begin{array}{l}\text { Pouteria caimito (Ruiz \& Pav.) } \\
\text { Radlk. }\end{array}$ & Leaf & Conical & Green & Absent \\
\hline Trotteria sp. & Mikania cf. biformis DC. & Leaf and stem & Conical & Green & Absent \\
\hline Trotteria sp. & Ocotea lobbii (Meisn.) Rohwer & Stem & Fusiform & Brown & Absent \\
\hline Trotteria sp. & Ocotea pulchella (Nees) Mez & Stem & No data & No data & No data \\
\hline $\begin{array}{l}\text { Trotteria sp. } \\
\text { Cecidophages }\end{array}$ & Eugenia copacabanensis Kiaersk. & Leaf & Fusiform & Red & Absent \\
\hline Clinodiplosis sp. & Atronium sp. & Leaf & Globoid & Green & Absent \\
\hline Thysanoptera & $\begin{array}{c}\text { Baccharis singularis (Vell.) G. M. } \\
\text { Barroso }\end{array}$ & Leaf and stem & No data & No data & No data \\
\hline $\begin{array}{c}\text { Acanthocheilla } \\
\text { sp. (Tingidae, } \\
\text { Hemiptera) }\end{array}$ & Mikania cf. biformis DC. & Leaf & Globoid & Green & Absent \\
\hline $\begin{array}{l}\text { Aphididae } \\
\text { (Hemiptera) }\end{array}$ & Mikania cf. biformis & Leaf & Globoid & Green & Absent \\
\hline $\begin{array}{l}\text { Coccidae } \\
\text { (Hemiptera) }\end{array}$ & Mikania cf. biformis & Leaf & Globoid & Green & Absent \\
\hline $\begin{array}{l}\text { Curculionidae } \\
\text { (Coleoptera) }\end{array}$ & Mikania cf. biformis & Leaf & Globoid & Green & Absent \\
\hline Lepidoptera & Baccharis speciosa DC. & Stem and bud & No data & No data & No data \\
\hline Lepidoptera & Piptocarpha cf. cinerea Baker & Leaf vein, stem and bud & Ovoid & Brown & Absent \\
\hline $\begin{array}{l}\text { Curculionidae } \\
\text { (Coleoptera) }\end{array}$ & Piptocarpha cf. cinerea & Leaf petiole, stem, bud & Globoid & Brown & Absent \\
\hline Lepidoptera & Piptocarpha cf. cinerea & Leaf petiole, stem, bud & Globoid & Brown & Absent \\
\hline Trypanea sp. & Porophyllum ruderale (Jack.) Cass. & Inflorescence & Fusiform & Green & Absent \\
\hline Curculionidae & Varronia curassavica Jacq. & Leaf vein & Fusiform & Green & Absent \\
\hline Coleoptera & Calophyllum brasiliense Cambess. & Leaf & Fusiform & Green & Absent \\
\hline Lepidoptera & $\begin{array}{c}\text { Elachyptera micranta (Cambess.) } \\
\text { A. C. Sm. }\end{array}$ & Leaf and bud & Conical & Green & Absent \\
\hline Curculionidae & Croton compressus Lam. & Inflorescence & Amorphous & Yellow & Present \\
\hline Curculionidae & Andira fraxinifolia Benth. & Leaf & Vermiform & Green & Absent \\
\hline Lepidoptera & Andira fraxinifolia & Leaf & Vermiform & Green & Absent \\
\hline Curculionidae & Andira fraxinifolia & Leaf & Vermiform & Green & Absent \\
\hline Coleoptera & Andira nitida Mart. ex Benth & Leaf & Lenticular & Green & Absent \\
\hline Lepidoptera & Inga sp. & Stem & Fusiform & $\begin{array}{l}\text { Green, } \\
\text { Brown }\end{array}$ & Present \\
\hline Curculionidae & Hyptis fasciculata Benth. & Leaf vein, petiole and stem & Fusiform & $\begin{array}{l}\text { Green } \\
\text { brown }\end{array}$ & Absent \\
\hline Cecidomyiidae & Ocotea notata (Nees \& Mart.) Mez & Leaf & Lenticular & $\begin{array}{l}\text { Green } \\
\text { yellow }\end{array}$ & Absent \\
\hline Hemiptera & Ocotea pulchella (Nees) Mez & Stem and bud & Fusiform & No data & Absent \\
\hline Thysanoptera & Struthanthus concinnus Mart. & Leaf and stem & Conical & Green & Absent \\
\hline Olcella sp. & Luehea divaricata Mart. & Stem & Globoid & Brown & Absent \\
\hline Curculionidae & Tibouchina trichopoda (DC.) Baill. & Stem & Fusiform & Brown & Absent \\
\hline Membracidae & $\begin{array}{c}\text { Campomanesia guaviroba (DC.) } \\
\text { Kiaersk. }\end{array}$ & Leaf vein & Globoid & No data & Absent \\
\hline
\end{tabular}


Table 5. Continued...

\begin{tabular}{|c|c|c|c|c|c|}
\hline \multirow{2}{*}{$\begin{array}{c}\text { Guilds } \\
\text { Inquilines }\end{array}$} & \multirow{2}{*}{ Host plant } & \multicolumn{4}{|c|}{ Gall characterization } \\
\hline & & Host organ & Shape & Color & Trichomes \\
\hline Curculionidae & Eugenia punicifolia (Kunt) DC. & Fruit & Globoid & $\begin{array}{c}\text { Green, } \\
\text { yellow, } \\
\text { Red }\end{array}$ & Absent \\
\hline Sciaridae & Eugenia speciosa Cambess. & Leaf & Conical & Yellow & Absent \\
\hline Thysanoptera & $\begin{array}{c}\text { Myrciaria floribunda (H.West ex } \\
\text { Willd.) O. Berg }\end{array}$ & Leaf & Globoid & Green & Present \\
\hline Stenoma annosa & $\begin{array}{c}\text { Neomitranthes obscura (DC.) N. J. } \\
\text { E. Silveira }\end{array}$ & Leaf & $\begin{array}{l}\text { Marginal } \\
\text { roll }\end{array}$ & Green & Absent \\
\hline S.annosa & Neomitranthes obscura & Bud & Conical & Green & Absent \\
\hline Lepidoptera & Guapira opposita (Vell.) Reitz & Leaf & Lenticular & Green & Absent \\
\hline Aphididae & Guapira opposita & Leaf & Globoid & Brown & Absent \\
\hline Lepidoptera & Guapira opposita & Flower peduncle & Globoid & $\begin{array}{c}\text { Green, } \\
\text { Brown, } \\
\text { red }\end{array}$ & Absent \\
\hline Lepidoptera & $\begin{array}{c}\text { Guapira pernambucensis (Casar.) } \\
\text { Lundell }\end{array}$ & Leaf & Lenticular & Green & Absent \\
\hline Coleoptera & $\begin{array}{l}\text { Ouratea cuspidata (A.St.-Hil.) } \\
\text { Engl. }\end{array}$ & Leaf & Conical & Brown & Absent \\
\hline Lepidoptera & Chaetocarpus myrsinites Baill. & Leaf & Lenticular & $\begin{array}{l}\text { Green, } \\
\text { Brown }\end{array}$ & Absent \\
\hline Muscomorpha & Securidaca sp. & Closed flower & Ovoid & Purple & Absent \\
\hline Lepidoptera & Coccoloba alnifolia Casar & Inflorescence & Globoid & $\begin{array}{l}\text { Green, } \\
\text { Yellow }\end{array}$ & Absent \\
\hline Lepidoptera & Paullinia sp. & $\begin{array}{l}\text { Leaf petiole, vein and } \\
\text { tendril }\end{array}$ & Fusiform & Green & Absent \\
\hline Sciaridae & Serjania communis Cambess. & Bud & Ovoid & Red & Absent \\
\hline Lepidoptera & Pouteria venosa (Mart.) Baehni & Leaf & Globoid & Green & Absent \\
\hline Coleoptera & $\begin{array}{c}\text { Manilkara subsericea (Mart.) } \\
\text { Dubard }\end{array}$ & Leaf & Lenticular & Green & Absent \\
\hline Curculionidae & $\begin{array}{l}\text { Aureliana fasciculata (Vell.) } \\
\text { Sendtn. }\end{array}$ & Leaf vein & Fusiform & Green & Absent \\
\hline $\begin{array}{l}\text { Haplothrips } \\
\text { gowdeyi }\end{array}$ & Stachytarpheta sp. & Inflorescence & Globoid & Green & Present \\
\hline
\end{tabular}

most frequent, being recorded in $38 \%$ and $36 \%$ of the galls with cecidophagous insects, while dipterans, hemipterans, and thysanopterans were recorded in $15 \%, 13 \%$, and $13 \%$ of the galls, respectively.

Only two cecidophages were identified to species: Stenoma annosa (Lepidoptera) and Haplothrips gowdeyi (Thysanoptera). Four were identified to genus: Clinodiplosis (Cecidomyiidae), Acanthocheilla (Tingidae), Trypanea (Tephritidae), and Olcella (Chloropidae). All other cecidophage records were identified to suprageneric categories.

\subsubsection{Inquiline guild}

Only eulophids (Hymenoptera) were considered inquilines in this revision, since their larvae were sedentary, exclusively phytophagous, coexisted with the gall-inducer and caused modification of gall tissues or stimulated the production of new tissues.

Modifications of gall tissues were reported in three gall morphotypes, one induced by Lopesia erythroxyli Rodrigues \& Maia, 2010 (Cecidomyiidae) on Erythroxylum ovalifolium Peyrs (Erythoxylaceae) (Figure 1), another induced by Stephomyia rotundifoliorum Maia, 1994 (Cecidomyiidae) on Eugenia astringens Camb. (Myrtaceae) (Figure 2), and the third induced by Myrciamyia maricaensis Maia, 1995 (Cecidomyiidae) on Myrcia ovata Camb. (Myrtaceae). The production of new tissues was reported in two gall morphotypes, one induced by Paulliniamyia ampla Maia, 2001 (Cecidomyiidae) on Paullinia weinmanniifolia Mart. (Sapindaceae) and the other induced by M. maricaensis on Myrcia ovata (Figures 3 and 4). None of these inquilines 

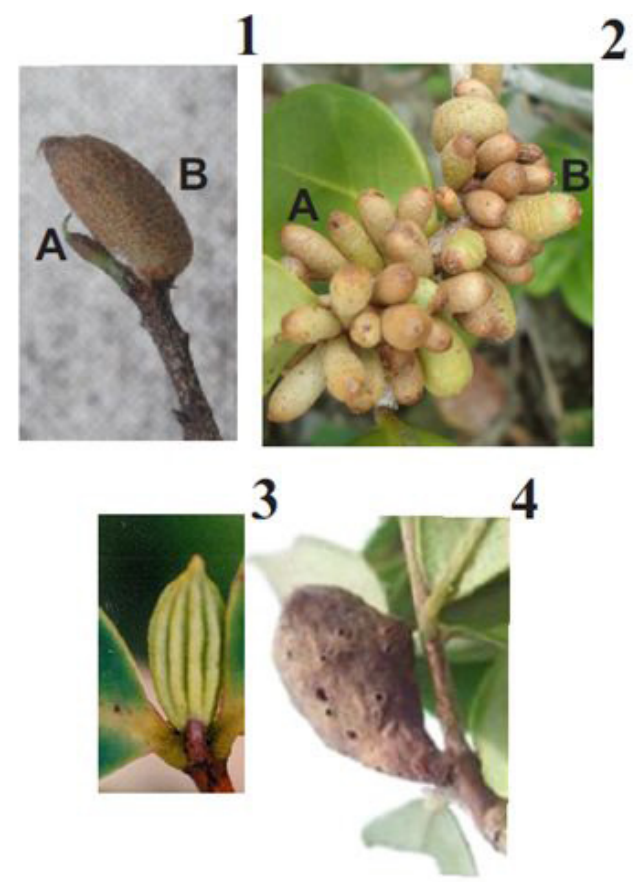

Figures 1-4. Galls of Cecidomyiidae. 1. Galls on Erythroxylum ovalifolium Peyrs. (Erythroxylaceae): (A) Gall induced by Lopesia erythroxyli Rodrigues and Maia, 2010; (B) The same gall modified by Eulophidae (Hymenoptera).2. Galls on Eugenia astringens Cambess.(Myrtaceae): (A) Gall induced by Stephomyia rotundifoliorum Maia, 1994; (B) The same gall modified by Eulophidae (Hymenoptera).3-4. Galls on Myrcia ovata Cambess.(Myrtaceae): 3. Gall induced by Myrciamyia maricaensis Maia, 1995, 4. The same gall modified by Eulophidae sp. 2 (Hymenoptera).

had a close phylogenetic relationship with the gall-inducer, and whenever they were present, the gall-inducers died.

Collectively, inquilines totaled five records (less than $8 \%$ ) in four gall morphotypes (less than $8 \%$ ) on plants of three families (16\%), four genera (11\%) and four species (9\%), all with a similar number of records (Table 5).

Inquilines were found mainly in bud galls, but also in leaf galls. They occurred in conical, ovoid, and cylindrical galls, with the first two being more frequent. They were obtained mainly from green galls, but also from brown galls, and all occurred in glabrous galls. Inquilines were identified only to the family level.

\subsubsection{Sucessors}

Leptothorax sp. (Formicidae) was reported as an inquiline of cylindrical galls on Eugenia hiemalis Camb. (Myrtaceae) by Maia (2001), but no gall modification was related to the presence of this ant. Furthermore, Leptothorax sp. could not be considered cecidophagous since it did not feed on gall tissues. Maia (2001) reported Leptothorax sp. as a successor in similar galls on Eugenia astringens Cambess. (Myrtaceae). These two cases are probably the same.

\subsubsection{Kleptoparasites}

Larvae of species of Contarinia, Resseliella, and Trotteria (Cecidomyiidae) were recorded in galls of other cecidomyiids, indicating a close phylogenetic relationship with the inducer since all belong to the same family. Whenever they were observed, they were the unique dwellers of galls, so the criterion of non-coexistence was fulfilled. Furthermore, these larvae showed low mobility and did not modify or stimulate production of new gall tissues. Therefore, they can be considered as kleptoparasites. Nevertheless, the criterion of food habit was not met, since their larvae were phytophagous and not omnivorous.

Tanaostigmatids, reported in galls of Meunieriella (Cecidomyiidae), were also considered kleptoparasites.

This guild totaled 13 records (20\%) in 12 gall morphotypes (23\%) on plants of eight families (33\%), 11 genera (30\%) and 12 species (27\%). All plant taxa had a similar number of records, so none of them can be highlighted as the most frequent (Table 5).

Kleptoparasites were represented by cecidomyiids (Diptera) and tanaostigmatids (Hymenoptera), which were obtained from leaf, stem, bud and fruit galls, being more frequent in the leaf galls (69\%). Kleptoparasites occurred in conical, globoid, fusiform, lenticular, ovoid, cylindrical and vermiform galls, but mainly in the first (33\%). Galls were green, yellow, brown, and red, but most (75\%) were green. All reports were in glabrous galls.

Two kleptoparasites were identified to species, Contarinia ubiquita and Trotteria quadridentata (Cecidomyiidae), seven to genera, Contarinia $(\mathrm{N}=1)$, Resseliella $(\mathrm{N}=2)$, and Trotteria $(\mathrm{N}=4)$, and four to family, Cecidomyiidae $(\mathrm{N}=3)$ and Tanaostigmatidae $(\mathrm{N}=1)$.

\subsection{Gall-inducing taxa and cecidophage, inquiline and kleptoparasite guilds}

Cecidophages, inquilines and kleptoparasites were recorded in 46 gall morphotypes induced by cecidomyiids (Diptera) and in two morphotypes induced by hemipterans. Cecidomyiid galls comprised all three of these guilds, while those of hemipterans sheltered only cecidophages. Some kleptoparasites and cecidophages were obtained from five gall morphotypes ( $9 \%$ of the total) whose inducers are still unknown.

Cecidophages, inquilines and kleptoparasites were associated with 16 species and 15 morphospecies of gallinducing cecidomyiids of 18 genera. Among these, galls induced by species of Lopesia Rübsaamen, 1908, hosted the greatest variety of guilds (cecidophages, inquilines, and kleptoparasites) and associated taxa (Coleoptera, Eulophidae, Cecidomyiidae, and Lepidoptera), followed by galls induced by species of Stephomyia Tavares, 1916, the galls of which sheltered inquilines (Eulophidae) and kleptoparasites (Trotteria sp.). Galls induced by species of Clinodiplosis Kieffer, 1894, hosted only cecidophages, as did those induced by species of Neolasioptera Felt, 1908, Asphondylia Loew, 1850, and Bruggmannia Tavares, 1906. Membracids, sciarids and curculionids were recorded in galls of Clinodiplosis, while thysanopterans and lepidopterans were recorded in galls of Neolasioptera. Representatives of Diptera (Cecidomyiidae and Trypanea sp.: Tephritidae) and Thysanoptera were obtained from galls of Asphondylia, 
whereas representatives of Hemiptera (Aphididae) and Lepidoptera were obtained from galls of Bruggmannia. The other gall midge genera sheltered a single guild and a single insect taxon.

Gall-inducing hemipterans were identified only to the level of order. Their galls hosted cecidomyiids, one of them identified to genus (Clinodiplosis sp.) and the other to family.

The new composition of these guilds allows the questions proposed at the beginning of this paper to be answered:

1) The most represented taxa among cecidophages were Lepidoptera and Coleoptera. Inquilines represented only Eulophidae (Hymenoptera) and kleptoparasites mainly Cecidomyiidae (Diptera). The richest taxa were not indicated, as most records were in suprageneric categories, without the discrimination of morphospecies;

2) Cecidomyiidae is the gall-inducing taxon with the richest fauna of inquilines, cecidophages and kleptoparasites;

3) Inquilines were reported in four gall morphotypes, four plant species, four genera and three families; cecidophages in 39 gall morphotypes, 35 plant species, 28 genera, and 20 families; and kleptoparasites in 12 gall morphotypes, eight plant families, 11 genera, and 12 species;

4) Asteraceae and Myrtaceae, Mikania (Asteraceae), Andira (Fabaceae), and Guapira (Nyctaginaceae), Mikania cf. biformis, Andira fraxinifolia (Fabaceae), Guapira opposita (Nyctaginaceae), Piptocarpha cf. cinerea, and Neomitranthes obscura (Myrtaceae) were the plant taxa with the richest cecidophage guild. No plant taxa were highlighted as sheltering the richest kleptoparasite guild or inquiline guild;

5) Cecidophages, inquilines and kleptoparasites were recorded mainly in leaf, green, and glabrous galls. The first occurred more frequently in globoid galls, the second in conical and ovoid galls, and the last in conical galls;

6) The taxonomic knowledge of these guilds remains poor, since most were identified to suprageneric categories, and only four to species level;

7) Biological data are necessary for the correct positioning of associated fauna into guilds.

\section{Discussion}

In this review, the cecidophage guild was easily determined based on the five criteria proposed by Luz and Mendonça-Júnior (2017). On the other hand, some conceptual problems were faced in determining inquilines and kleptoparasites. According to these authors, inquilines have a close phylogenetic relationship with the gall-inducing species. This criterion was not met, but the other four (food habit, coexistence with the inducer, modification of gall tissues or production of new tissues, and mobility) were fulfilled. Regarding food habits, kleptoparasites are defined as omnivorous, which is a criterion not met by gall midges, although they met the other criteria. The use of these five criteria is only possible when biological data are known, which demands long-term studies.

The presence of cecidophages, inquilines and kleptoparasites can indirectly cause the death of the inducer, which can be important for population dynamics of gallinducing species. However, this effect was little discussed in Luz and Mendonça-Júnior (2017). Nevertheless, these guilds should be considered since their presence can be an important mortality factor, as indicated by Maia, 2001.

Cecidophages were represented mainly by lepidopterans and coleopterans. These two insect orders were also indicated as the most represented among the secondary fauna inhabiting galls of cynipids (Hymenoptera) (Giannetti et al., 2019).

Cecidophage and kleptoparasite guilds were obtained mainly from leaf, green, and glabrous galls. These are the predominant features of insect galls in Brazilian restingas (Maia, 2001; Maia et al., 2008; Rodrigues et al., 2014), suggesting that guild frequency is related to resource availability. Nevertheless, other gall features were predominant for inquilines, but the number of records was too low to make generalizations. Most records were for cecidomyiid galls, the most diverse, abundant and frequent gall-inducing taxon in restingas (Maia, 2001; Maia et al., 2008; Rodrigues et al., 2014).

Asteraceae and Myrtaceae hosted the greatest richness and frequency of cecidophages, which was expected since they are the plant families with the greatest gall richness in restingas (Maia, 2013). Both families are well represented in this ecosystem (Lourenço and Barbosa, 2012; Melo-Júnior and Boeger, 2018). No plant family stood out as exhibiting the greatest number of kleptoparasite or inquiline records since both guilds were similarly distributed among different families. However, their records are also few, so new and broad studies are likely to modify this scenario.

The plant genera and species with the highest number of cecidophages were not necessarily those that hosted the greatest number of galls, since only Mikania and Guapira opposita (Nyctaginaceae) have been been cited as super host taxa (Maia and Oliveira, 2010; Maia, 2013; Rodrigues et al., 2014). Thus, cecidophage richness appears not to be related to gall richness. In fact, some galls can be more attractive than others, probably due to their own morphological and chemical features.

The high frequency of cecidophagy found here reveals the importance of gall-inducing insects as ecosystem engineers in restingas. Inducers do not merely provide habitat for specialists, but can also influence the structure of communities that do not directly interact with galls, as Wetzel et al. (2016) has shown. They can have significant impacts on the herbivore community not only by changing plant morphology, but also by altering host quality and modifying plant-induced responses to subsequent herbivory (Uesugi et al., 2016).

The taxonomic knowledge of cecidophage, kleptoparasite, and inquiline guilds remains still poor. The scarcity of identified species, for example, does not allow discussions to be made about their specificity.

A more complete review of guild richness, including also parasitoids, predators and symbionts, can contribute 
to revealing the importance of associated faunas for gall systems. However, in order to know the composition of each guild, specific identification is essential, as well as the correct categorization of inquilines, kleptoparasites and cecidophages, which depends on taxonomical and biological data, respectively.

\section{Conclusions}

Although cecidophages were not previously recorded in insect gall inventories in Brazilian restingas, they are actually frequent. Kleptoparasites are also present. Both of these guilds are formally reported here for the first time in this ecosystem. On the other hand, the frequency and diversity of inquilines are low, differing from literature data. These new records resulted from recategorization among guilds based on literature data and biological observations, following criteria proposed by Luz and Mendonça-Júnior (2017).

Although the kleptoparasites found did not fulfill the food habit criterion, and the inquilines did not satisfy the phylogenetic relationship criterion, both guilds could be easily established based on the other four criteria. In my opinion, future studies about insect galls and associated fauna should adopt these criteria to avoid misinterpretation and improve knowledge about these guilds in Brazil.

\section{Acknowledgements}

This research was supported by the Conselho Nacional de Desenvolvimento Científico e Tecnológico (Proc. 301481/2017-2).

\section{References}

ANSALONI, S., SALMAZO, J.R. and URSO-GUIMARÃES, M.V., 2018. Entomogen galls in a Seasonal Semideciduous Forest area in Sorocaba, Southeast of São Paulo State, Brazil. Biota Neotropica, vol. 18, no. 4, pp. e20180523. http://dx.doi.org/10.1590/16760611-bn-2018-0523.

ARAÚJO, W.S., GOMES-KLEIN, V.L. and SANTOS, B.B., 2007. Galhas entomógenas associadas à vegetação do Parque Estadual da Serra dos Pireneus, Pirenópolis, Goiás, Brasil. Revista Brasileira de Biociências, vol. 5, suppl. 1, pp. 45-47.

ARAÚJO, W.S., SANTOS, B.B. and GOMES-KLEIN, V.L., 2011. Insect galls from Serra dos Pirineus, GO, Brazil. Biota Neotropica, vol. 11, no. 2, pp. 357-365. http://dx.doi.org/10.1590/S167606032011000200034.

ASCENDINO, S. and MAIA, V.C., 2018. Insects galls of Pantanal areas in the State of Mato Grosso do Sul, Brazil: characterization and occurrence. Anais da Academia Brasileira de Ciências, vol. 90, no. 2, pp. 1543-1564. http://dx.doi.org/10.1590/00013765201820170535. PMid:29791563.

BERGAMINI, B.A.R., BERGAMINI, L.L., SANTOS, B.B. and ARAÚJO, W.S., 2017. Occurrence and characterization of insect galls in the Floresta Nacional de Silvania. Papéis Avulsos de Zoologia, vol. 57, no. 32, pp. 413-431. http://dx.doi.org/10.11606/00311049.2017.57.32.

BREGONCI, J.M., POLYCARPO, P.V. and MAIA, V.C., 2010. Galhas de insetos do Parque Estadual Paulo César Vinha (Guarapari,
ES, Brasil). Biota Neotropica, vol. 10, no. 1, pp. 265-274. http:// dx.doi.org/10.1590/S1676-06032010000100023.

BRITO, G.P., COSTA, E.C., CARVALHO-FERNANDES, S.P. and SANTOSSILVA, J., 2018. Riqueza de galhas de insetos em áreas de Caatinga com diferentes graus de antropização do estado da Bahia, Brasil. Iheringia. Série Zoologia, vol. 108, e2018003. http:// dx.doi.org/10.1590/1678-4766e2018003.

CARVALHO, A.N. and MOTTA, J.S., 2018. Ocorrência e caracterização de galhas entomógenas em um fragmento florestal em estágio de sucessão ecológica na Amazônia. EntomoBrasilis, vol. 11, no. 2, pp. 118-123. http://dx.doi.org/10.12741/ebrasilis.v11i2.786.

CARVALHO-FERNANDES, S.P., ALMEIDA-CORTEZ, J. and FERREIRA, A.L.N., 2012. Riqueza de galhas entomógenas em áreas antropizadas e preservadas de caatinga. Revista Árvore, vol. 36, no. 2, pp. 269-277. http://dx.doi.org/10.1590/S010067622012000200008 .

CARVALHO-FERNANDES, S.P., ASCENDINO, S., MAIA, V.C. and COURI, M.S., 2016. Diversity of insect galls associated with coastal shrub vegetation in Rio de Janeiro, Brazil. Anais da Academia Brasileira de Ciências, vol. 88, no. 3, pp. 1407-1418. http:// dx.doi.org/10.1590/0001-3765201620150658. PMid:27627066.

COSTA, E.C., CARVALHO-FERNANDES, S.P. and SANTOS-SILVA, J., 2014. Galhas entomógenas associadas à Leguminosae do entorno do riacho Jatobá, Caetité, Bahia, Brasil. Revista Brasileira de Biociências, vol. 12, no. 2, pp. 115-120.

FERNANDES, G.W.A., TAMEIRÃO NETO, E. and MARTINS, R.P., 1988. Ocorrência e caracterização de galhas entomógenas do Campus Pampulha da Universidade Federal de Minas Gerais. Revista Brasileira de Zoologia, vol. 5, no. 1, pp. 11-29. http:// dx.doi.org/10.1590/S0101-81751988000100002.

FERRAZ, F.F.F. and MONTEIRO, R.F., 2003. Complex interactions envolving a gall midge Myrciamyia maricaensis Maia (Diptera, Cecidomyiidae), phytophagous modifiers and parasitoids. Revista Brasileira de Zoologia, vol. 20, no. 3, pp. 433-437. http://dx.doi. org/10.1590/S0101-81752003000300011.

FLOR, I.C., FLOR, J.C.R. and FURTADO, P.S.N., 2018. Insect galls of the Floresta da Cicuta (Volta Redonda, RJ, Brazil). Papéis Avulsos de Zoologia, vol. 58, e20185824.

GIANNETTI, D., CASTRACANI, C., SPOTTI, F.A., MORI, A. and GRASSO, A., 2019. Gall-colonizing ants and their role as plant defenders: from "bad job" to "useful service". Insects, vol. 10, no. 11, pp. 392. http://dx.doi.org/10.3390/insects10110392. PMid:31698832.

GODFRAY, H.C.J., 1994. Parasitoids: behavioral and evolutionary ecology. Princeton: Princeton University Press, 473 p. http:// dx.doi.org/10.1515/9780691207025.

LIMA, V.P. and CALADO, D., 2018. Morphological characterization of insect galls and new records of associated invertebrates in a Cerrado area in Bahia State, Brazil. Brazilian Journal of Biology = Revista Brasileira de Biologia, vol. 78, no. 4, pp. 636-643. http:// dx.doi.org/10.1590/1519-6984.169502. PMid:29319753.

LOURENÇO, A.R. and BARBOSA, M.R.V., 2012. Myrtaceae em restingas no limite norte de distribuição da Mata Atlântica, Brasil. Rodriguésia, vol. 63, no. 2, pp. 373-393. http://dx.doi. org/10.1590/S2175-78602012000200011.

LUZ, F.A. and MENDONÇA-JÚNIOR, M.S., 2017. Guilds in insect galls: who is who. The Florida Entomologist, vol. 102, no. 1, pp. 207-210. http://dx.doi.org/10.1653/024.102.0133.

MAIA, V.C. and CARVALHO-FERNANDES, S.P., 2016. Insect galls of a protected remnant of the Atlantic Forest tableland from Rio de Janeiro State (Brazil). Revista Brasileira de Entomologia, vol. 60, no. 1, pp. 40-56. http://dx.doi.org/10.1016/j. rbe.2015.09.001. 
MAIA, V.C. and FERNANDES, G.W., 2004. Insect galls from Serra de São José (Tiradentes, MG, Brazil). Brazilian Journal of Biology = Revista Brasileira de Biologia, vol. 64, no. 3A, pp. 423-445. http:// dx.doi.org/10.1590/S1519-69842004000300007. PMid:15622841.

MAIA, V.C. and MASCARENHAS, B., 2017. Insect galls of the Parque Nacional do Itatiaia (Southeast Region, Brazil). Anais da Academia Brasileira de Ciências, vol. 89, no. 1, suppl., pp. 505-575. http:// dx.doi.org/10.1590/0001-3765201720160877. PMid:28562824.

MAIA, V.C. and OLIVEIRA, J.C., 2010. Galhas de insetos da Reserva Biológica Estadual da Praia do Sul (Ilha Grande, Angra dos Reis, RJ). Biota Neotropica, vol. 10, no. 4, pp. 227-238. http://dx.doi. org/10.1590/S1676-06032010000400028.

MAIA, V.C. and SIQUEIRA, E.S., 2020. Insect galls of the Reserva Biológica União, Rio de Janeiro, Brazil. Biota Neotropica, vol. 20, no. 1, pp. e20190758. http://dx.doi.org/10.1590/1676-0611-bn-2019-0758.

MAIA, V.C. and SOUZA, M.C., 2013. Insect galls of the xeric vegetation of Ilha do Cabo Frio (Arraial do Cabo, RJ, Brazil). Biota Neotropica, vol. 13, no. 3, pp. 278-288. http://dx.doi.org/10.1590/S167606032013000300030 .

MAIA, V.C., 1995. Chaves para classificação de galhas de Cecidomyiidae (Diptera) em Myrtaceae na restinga da Barra de Maricá, Rio de Janeiro. Revista Brasileira de Zoologia, vol. 12, no. 4, pp. 10091013. http://dx.doi.org/10.1590/S0101-81751995000400028.

MAIA, V.C., 2001. The gall midges (Diptera, Cecidomyiidae) from three restingas of Rio de Janeiro State, Brazil. Revista Brasileira de Zoologia, vol. 18, no. 2, pp. 583-629. http://dx.doi.org/10.1590/ S0101-81752001000200028.

MAIA, V.C., 2011. Characterization of insect galls, gall makers, and associated fauna of Platô Bacaba (Porto de Trombetas, Pará, Brazil). Biota Neotropica, vol. 11, no. 4, pp. 37-53. http://dx.doi. org/10.1590/S1676-06032011000400003.

MAIA, V.C., 2013. Insect galls of São Tomé das Letras (MG, Brazil). Biota Neotropica, vol. 13, no. 4, pp. 164-189. http://dx.doi. org/10.1590/S1676-06032013000400017.

MAIA, V.C., 2014. Insect galls of Itamonte (Minas Gerais, Brazil): characterization and occurrence. Biota Neotropica, vol. 14, no. 1, pp. 1-17. http://dx.doi.org/10.1590/S1676-06033839.

MAIA, V.C., AZEVEDO, M.A.P. and COURI, M.S., 2002. New contribution to the knowledge of the gall midges (Diptera, Cecidomyiidae) from the restinga of Barra de Maricá (Rio de Janeiro, Brazil). Studia Dipterologica, vol. 9, pp. 447-452.

MAIA, V.C. and AZEVEDO, M.A.P., 2009. Micro-himenópteros associados com galhas de Cecidomyiidae (Diptera) em restingas do estado do Rio de Janeiro. Biota Neotropica, vol. 9, no. 2, pp. 151-164. http://dx.doi.org/10.1590/S1676-06032009000200015.

MAIA, V.C., CARDOSO, J.L.T. and BRAGA, J.M.A., 2014. Insect galls from Atlantic Forest areas of Santa Teresa, Espírito Santo, Brazil: characterization and occurrence. Boletim do Museu de Biologia Mello Leitão, vol. 33, pp. 47-129.

MAIA, V.C., MAGENTA, M.A.G. and MARTINS, S.E., 2008. Ocorrência e caracterização de galhas de insetos em áreas de restinga de Bertioga (São Paulo, Brasil). Biota Neotropica, vol. 8, no. 1, pp. 167-197. http://dx.doi.org/10.1590/S1676-06032008000100020.

MANI, M.S., 1964. Ecology of plant galls. The Hague: Dr. W. Junk. http://dx.doi.org/10.1007/978-94-017-6230-4.

MELO-JÚNIOR, J.C.F. and BOEGER, M.R.T., 2018. Riqueza e estrutura de uma comunidade vegetal de dunas na planície costeira de Santa Catarina. Iheringia. Série Botânica, vol. 73, no. 3, pp. 290-297. http://dx.doi.org/10.21826/24468231201873306.

MOUND, L.A. and MORRIS, D.C., 2000. Inquilines or kleptoparasites? New phlaeothripine Thysanoptera associated with domicile- building thrips on Acacia trees. Australian Journal of Entomology, vol. 39, no. 3, pp. 130-137. http://dx.doi.org/10.1046/j.14406055.2000.00165.x.

RAMAMURTHY, V.V., 2007. Faunistic, ecological, biogeographical and phylogenetic aspects of Coleoptera as gall-inducers and associates in plant galls in the Orient and eastern Palearctic. Oriental Insects, vol. 41, no. 1, pp. 93-119. http://dx.doi.org/10. 1080/00305316.2007.10417501.

REDFERN, M. and ASKEW, R.R., 1992. Plant galls. Slough: Richmond Publishing. Naturalists' Handbooks, no. 17.

RIBEIRO, A.N., BALBI, M.I.P.A. and URSO-GUIMARÃES, M.V., 2019. Characterization of insect galls from a vegetation area in Altinópolis, São Paulo State, Brazil. Papéis Avulsos de Zoologia, vol. 59, e20195904. http://dx.doi.org/10.11606/18070205/2019.59.04.

RODRIGUES, A.R., MAIA, V.C. and COURI, M.S., 2014. Insect galls of restinga areas of Ilha da Marambaia, Rio de Janeiro, Brazil. Revista Brasileira de Entomologia, vol. 58, no. 2, pp. 173-197. http://dx.doi.org/10.1590/S0085-56262014000200010.

SAITO, V.S. and URSO-GUIMARÃES, M.V., 2012. Characterization of galls, insect galls and associated fauna of Ecological Station of Jataí (Luiz Antônio, SP). Biota Neotropica, vol. 12, no. 3, pp. 1-9. http://dx.doi.org/10.1590/S167606032012000300011.

SANTOS, B.B., FERREIRA, H.D. and ARAÚJO, W.S., 2010. Ocorrência e caracterização de galhas entomógenas em uma área de floresta estacional semidecídua em Goiânia, Goiás, Brasil. Acta Botanica Brasílica, vol. 24, no. 1, pp. 243-249. http://dx.doi.org/10.1590/ S0102-33062010000100026.

SANTOS, I.S., LIMA, V.P., SOARES, E.K.S., PAULA, M. and CALADO, D.C., 2018. Insect galls in three species of Copaifera L. (Leguminosae, Caesalpinioideae) occurring sympatrically in a Cerrado area (Bahia, Brazil). Biota Neotropica, vol. 18, no. 1, pp. e20170356. http://dx.doi.org/10.1590/1676-0611-bn-2017-0356.

SANTOS, J.C., RIBEIRO, B.A., SILVA, T.M. and ARAUJO, W.S., 2012. Galhas de insetos em uma área de cerrado sentido restrito na região semi-urbana de Caldas Novas (Goiás, Brasil). Revista Brasileira de Biociências, vol. 10, no. 4, pp. 439-445.

SILVA, A.R.F., NOGUEIRA, R.M., COSTA, E.C., CARVALHOFERNANDES, S.P. and SANTOS-SILVA, J., 2018a. Occurrence and characterization of entomogenic galls in an area of Cerrado sensu stricto and Gallery forest of the state of Bahia, Brazil. Anais da Academia Brasileira de Ciências, vol. 90, no. 3, pp. 29032919. http://dx.doi.org/10.1590/0001-3765201820170522. PMid:30304224.

SILVA, E.C., SANTOS, B.B. and ARAÚJO, W.S., 2018b. Insect gall occurrence in savanna and forest remnant sites of Hidrolândia, GO, Brazil Central. Papéis Avulsos de Zoologia, vol. 58, e20185804. http://dx.doi.org/10.11606/1807-0205/2018.58.04.

UESUGI, A., MORRELL, K., POELMAN, E.H., RAAIJMAKERS, C.E. and KESSLER, E., 2016. Modification of plant-induced responses by an insect ecosystem engineer influences the colonization behaviour of subsequent shelter-users. Journal of Ecology, vol. 104, no. 4, pp. 1096-1105. http://dx.doi.org/10.1111/1365-2745.12587.

URSO-GUIMARÃES, M.V., SCARELI-SANTOS, C. and BONIFÁCIO-SILVA, A.C., 2003. Occurrence and characterization of entomogen galls in plants from natural vegetation areas in Delfinópolis, MG, Brazil. Brazilian Journal of Biology = Revista Brasileira de Biologia, vol. 63, no. 4, pp. 705-715. http://dx.doi.org/10.1590/ S1519-69842003000400018. PMid:15029382.

VIEIRA, L.G., NOGUEIRA, R.M., COSTA, E.C., CARVALHO-FERNANDES, S.P. and SILVA, J.S., 2018. Insect galls in Rupestrian field and Cerrado stricto sensu vegetation in Caetité, Bahia, Brazil. 
Biota Neotropica, vol. 18, no. 2, pp. e20170402. http://dx.doi. org/10.1590/1676-0611-bn-2017-0402.

WETZEL, W.C., SCREEN, R.M., LI, I., MCKENZIE, J., PHILLIPS, K.A., CRUZ, M., ZHANG, W., GREENE, A., LEE, E., SINGH, N., TRAN, C. and YANG, L.H., 2016. Ecosystem engineering by a gall-forming wasp indirectly suppresses diversity and density of herbivores on oak trees. Ecology, vol. 97, no. 2, pp. 427-438. http://dx.doi. org/10.1890/15-1347.1. PMid:27145617. 